\title{
Prevalence and Distribution of HPV Genotypes in 1387 Women with Cervical Intraepithelial Neoplasia 2/3 in Shanxi Province, China
}

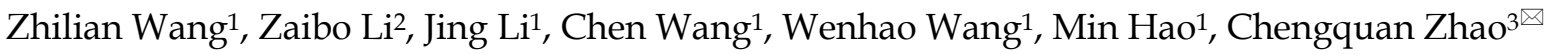 \\ 1. Department of Obstetrics and Gynecology, Second Hospital of Shanxi Medical University, Taiyuan, China. \\ 2. Department of Pathology, Ohio State Wexner Medical Center, Columbus, OH 43210, USA. \\ 3. Department of Pathology, Magee-Womens Hospital, University of Pittsburgh Medical Center. 300 Halket St, Pittsburgh, PA, 15213, USA.
}

$\square$ Corresponding author: Min Hao, MD. Department of Obstetrics and Gynecology, The Second Hospital of Shanxi Medical University, 382 Wuyi Rd, Taiyuan, China. Email :2yuanhaomin@163.com; Chengquan Zhao, Department of Pathology, Magee-Womens Hospital, University of Pittsburgh Medical Center. 300 Halket St, Pittsburgh, PA, 15213, USA.

(C) Ivyspring International Publisher. This is an open access article distributed under the terms of the Creative Commons Attribution (CC BY-NC) license (https://creativecommons.org/licenses/by-nc/4.0/). See http://ivyspring.com/terms for full terms and conditions.

Received: 2018.02.19; Accepted: 2018.06.10; Published: 2018.07.16

\begin{abstract}
Objective: The distribution of human papillomavirus (HPV) genotypes varies by countries and regions. Data regarding HPV genotype distribution for women with cervical intraepithelial neoplasia 2/3 (CIN2/3) are still limited. This study aimed to investigate the distribution of HPV genotypes for women with CIN2/3 in Shanxi province, China.

Methods: Prior HPV testing/genotyping resulted from women with histologic diagnosis of CIN2/3 were retrospectively collected between August 2011 and August 2016.

Results: A total of 1387 women with a diagnosis of CIN2/3 were included in this study. The average age of the women was 45.7 years and the average time period from HPV testing to histological diagnosis was 0.4 months. The overall high-risk HPV (hrHPV) positive rate was $91.6 \%$. In all tested women, the most commonly detected hrHPV genotype was HPV16 (59.3\%) followed by HPV58 (14.4\%) and HPV33 (10.0\%), while HPV18 was in the $6^{\text {th }}$ position (6.0\%).

Conclusions: This is one of the largest studies of HPV genotyping for women with CIN2/3 in China. The prevalence and distribution of HPV genotypes in these Chinese women differed from those in women from Western countries. Our results provide important information for developing specific HPV vaccines for Chinese women.
\end{abstract}

Key words: HPV genotyping, Neoplasia 2/3, Prevalence

\section{Introduction}

Cervical cancer is the fourth most common cancer among women worldwide in terms of morbidity and mortality. More than $85 \%$ of cervical cancers occur in the developing countries. ${ }^{1}$ China accounts for $14 \%$ of the world's annual incidence of cervical cancer, and hence it remains an important public health problem in China. ${ }^{2,3}$ The incidence of cervical cancer in Shanxi Province, China is strikingly high. ${ }^{4}$ Persistent infection of high-risk Human Papillomavirus (hrHPV) infection is critical for the development of almost all cervical cancers and precancerous lesions. ${ }^{5} \mathrm{~A}$ meta-analysis of 48 studies showed that HPV16 and HPV18 are the most prevalent genotypes worldwide. ${ }^{6}$ It has been shown that the distribution of HPV genotypes in cervical cancers varies by countries and regions. ${ }^{7}$ China is a large country with varied socioeconomic conditions among different regions, and HPV infections differ accordingly. ${ }^{8}$ A large study from China, which included 51,345 women, has demonstrated three most common hrHPV types were HPV52, 16, and 58, while HPV18 was only the $6^{\text {th }}$ most common type. ${ }^{9}$

HPV vaccination is one of the most important measures to prevent HPV infection. ${ }^{10}$ However, all 
currently available vaccines were developed based on the epidemiological data from Western countries, and as a result, target HPV16, HPV18 and several other commonly identified subtypes in Western countries. ${ }^{11}$, 12, 13 Implementation of these vaccines in China may not be able to achieve the desired preventive efficacy. Therefore, in order to develop HPV vaccines specific for Chinese women, it is necessary to investigate HPV genotype distributions in China.

\section{Materials and Methods}

\section{Study participants}

The cases with histological diagnosis of CIN2/3 rendered between August 2011 and August 2016 were retrospectively searched in the patient information system at Second Hospital of Shanxi Medical University (SHSMU). Only the cases with prior HPV genotyping results in the preceding 6 months were included in the study and analyzed. Other clinical information, including age and clinical history were also collected. Histopathologic diagnoses were reported on samples including endocervical curettage specimens, cervical biopsies, loop electrosurgical excision procedures or cold-knife conization. The Second Hospital of Shanxi Medical University is a large, integrated, public health system located in the capital of Shanxi, which accommodates patients from all over Shanxi province.

\section{HPV Geno-array test}

HPV detection and genotyping was tested using HybriMax HPV Geno-Array kit (Hybribio Biotechnology Limited Corp., Chaozhou, China) at the Second Hospital of Shanxi Medical University laboratory. The HPV Geno-Array can determine 21 HPV types, including $15 \mathrm{hrHPV}$ types(16, 18, 31, 33, $35,39,45,51,52,53,56,58,59,66$, and 68) and 6 low risk HPV (lrHPV) types (6, 11, 41, 42, 44, and CP8304).Test was performed according to the manufacturer's instructions. ${ }^{14}$ Briefly, PCR was performed in a $25 \mu \mathrm{L}$ reaction mixture containing $5 \mu \mathrm{L}$ extracted DNA, $0.75 \mu \mathrm{L}$ DNA Taq polymerase and 19.25 $\mu \mathrm{L}$ PCR-mix solution containing MY09/11 primer system. The PCR protocol was: denaturing at $95^{\circ} \mathrm{C}$ for $9 \mathrm{~min}$, followed by 40 cycles of $20 \mathrm{~s}$ at $95^{\circ} \mathrm{C}$, 30 s at $55^{\circ} \mathrm{C}$, and 30 s at $72{ }^{\circ} \mathrm{C}$, at last a finally extension at $72{ }^{\circ} \mathrm{C}$ for $5 \mathrm{~min}$. A positive control and a negative control were run in each PCR analysis process to control for possible contamination and accuracy. The kit can simultaneously identify 21 HPV genotypes: adding NBT/BCIP solution to display the results, a positive result was indicated by a clearly visible indigo dot. The HPV-genotype result was determined by the position of the HPV-genotype probes on the microarray chip. The kit can simultaneously identify
21 HPV genotypes: adding NBT/BCIP solution to display the results, a positive result was indicated by a clearly visible indigo dot. The HPV-genotype result was determined by the position of the HPV-genotype probes on the microarray chip. Multiple dots indicated multiple infections. ${ }^{15}$

\section{Colposcopy and cervical histopathologic diagnosis}

Colposcopy exams were performed by specialists according to a standardized protocol. During the exam, all visually abnormal areas were biopsied. The squamocolumnar junction was biopsied ("random biopsy") if no visible lesion identified. Endocervical curettage (ECC) was then performed according to the aforementioned indications.

\section{Statistical analysis}

The Pearson chi-square test was used for statistical analysis, which was conducted with SAS 9.1 software (SAS Institute, Cary, North Carolina). A P value less than. 05 was considered statistically significant.

\section{Results}

\section{Overall HPV prevalence}

A total of 1,387 CIN2/3 cases with HPV genotyping results within 6 months prior to histological diagnosis were included in the study. The overall hrHPV positive rate was 91.9\% (1274/1387). The average time period from HPV testing to histological diagnosis was 0.4 months (range: 0.1-6 months) and the average age was 45.7 years (range: 21-83 years).

\section{Distribution of single and multiple hrHPV infections}

Single hrHPV infection was identified in $65.0 \%$ of all women (902/1,387), accounting for $71.0 \%$ $(902 / 1,271)$ of hrHPV positive cases. Infection with multiple hrHPV types was identified in $26.6 \%$ of all women, accounting for $29.0 \%$ of hrHPV positive cases. Among the women infected with multiple hrHPV types, infection of two genotypes was most commonly observed with an overall prevalence rate of $17.5 \%$. Infection with three and more hrHPV types were found in 99 women with an overall prevalence rate of $7.1 \%$ (Table 1 ).

\section{HrHPV genotype distribution}

The most commonly detected hrHPV genotype was HPV16 (59.3\%), followed by HPV58 (14.4\%) and HPV33 $(10.0 \%)$, while HPV18 was in the $6^{\text {th }}$ position $(6.0 \%)$ (Table 2). 
Table 1. 1387 cases with genotyping results 6 months before $\mathrm{CIN} 2 / 3$ diagnosis

\begin{tabular}{lll}
\hline HPV infection & Positive Case & $\%$ \\
\hline 1 HrHPV only & 902 & 65.0 \\
2 HrHPV only & 244 & 17.5 \\
$\geq 3$ HrHPV only & 96 & 6.9 \\
Any mixed Hr and LrHPV & 32 & 2.3 \\
LrHPV only & 10 & 0.7 \\
Negative (no both Hr and & 103 & 7.4 \\
Lr HPV & & $100 \%$ \\
Total & 1387 & \\
Abbreviations: HrHPV, high-risk human papillomavirus; LrHPV, low-risk human \\
papillomavirus number; CIN2/3, cervical intraepithelial neoplasia 2/3.
\end{tabular}

Table 2. HPV genotypes screening test results 6 months before CIN2/3 diagnosis (1387 cases)

\begin{tabular}{lll}
\hline HPV type & Case No & Percentage \\
\hline HrHPV & & \\
16 & 823 & 59.3 \\
18 & 79 & 6.00 \\
31 & 105 & 7.6 \\
33 & 139 & 10.0 \\
35 & 31 & 2.2 \\
39 & 47 & 3.4 \\
45 & 25 & 1.8 \\
51 & 67 & 4.8 \\
52 & 98 & 7.1 \\
53 & 60 & 4.3 \\
56 & 35 & 2.5 \\
58 & 200 & 14.4 \\
59 & 27 & 1.9 \\
66 & 22 & 1.6 \\
68 & 0 & 0.00 \\
LrHPV & & \\
11 & 23 & 1.7 \\
42 & 12 & 0.9 \\
81 & 14 & 1.0 \\
6 & 7 & 0.5 \\
41 & 3 & 0.2 \\
44 & 4 & 0.3 \\
\hline Abbreviations: HrHPV, high-risk human papillomavirus; LrHPV, low-risk human \\
papillomavirus number; CIN2/3, cervical intraepithelial neoplasia 2/3.No, \\
number. & & \\
& &
\end{tabular}

\section{HrHPV infection among women of different Ages}

All the 1,387 cases were divided into three age groups ( $<40$ years, $40-49$ years, and $\geq 50$ years). The overall prevalence of hrHPV was $93.2 \%$ in women $<40$ years, $90.0 \%$ in women $40-49$ years, and $92.2 \%$ in women $\geq 50$ years. HrHPV positive rates showed no significant difference among these three age groups $(p=0.1978)$. Additionally, the positive rates of HPV16 in the three groups were also similar and were $59.2 \%$ in women $<40$ years, $58.2 \%$ in women $40-49$ years, and $60.6 \%$ in women $\geq 50$ years respectively $(p=0.7273$ ).

\section{Discussion}

Epidemiological studies have suggested a substantial difference in the prevalence and genotype distribution of hrHPV infection among women from different countries. 16,17,18,19,20 Several large studies from China have demonstrated hrHPV prevalence rates in general population range from $9.9 \%$ to $21.1 \%$. 9, 21, 22 However, studies of hrHPV infection in Chinese women with CIN2/3 were lacking. A study has reported that approximately $82.4 \%$ of cervical precancerous lesions and $92 \%$ of cervical invasive carcinoma are HPV-positive ${ }^{23}$. Zhao et al. reported that all 12 cases of cervical cancer and 31 cases of CIN3, and $95.3 \%$ of CIN2 (43 cases) were hrHPV positive. ${ }^{24}$ To our knowledge, the current study is one of the largest studies to investigate hrHPV prevalence in Chinese women with CIN2/3, demonstrating that $91.6 \%$ of these women were hrHPV positive.

In consistent with previous reports, ${ }^{25}, 26$ single HPV type infection is the most common infection in the current study, followed by the HPV infection with two types. Infection with 3 or more HPV types is rare. It remains exclusive whether HPV infection with multiple types is linked to occurrence or progression of cervical cancerous/precancerous lesions. One study suggested HPV infection with multiple types has increased risk for cervical cancerous/ precancerous lesions ${ }^{27}$, while other studies did not show any increased risk for HPV infection with multiple types. ${ }^{28,}{ }^{29}$ Further studies are still warranted to elucidate the role of infection with multiple HPV types in the development of cervical cancer.

HPV genotyping results for Chinese women with CIN2/3 are limited. The current study demonstrated the most commonly detected hrHPV genotype was HPV16 (59.3\%), followed by HPV58 $(14.4 \%)$ and HPV33 (10.0\%), while HPV18 was only in the 6 th position $(6.0 \%)$. HPV16 was the predominant type among women with CIN2/3 in this study, consistent with previous studies from Western countries. ${ }^{30,31}$ However, the $2^{\text {nd }}$ and $3^{\text {rd }}$ most commonly detected types in current study were HPV58 and HPV33, which are different from the results observed in women from Western countries. ${ }^{32}$, 33, 34, 35 HPV 16, HPV 33, and HPV 52 have been reported to be the most prevalent HPV types in Chinese women, however, the distribution of HPV genotype has been shown to be different from regions to regions in China. ${ }^{9,36,37,38} \mathrm{~A}$ study of 6723 subjects in four large cities (Beijing, Shanghai, Shenzhen, and Shenyang) and four rural areas (Shanxi: Xiangyuan, Yangcheng, Henan Xinmi, and Xinjiang: Hotan) in China demonstrated HPV16 is the most common type, followed by HPV58 and HPV18. ${ }^{39}$ However, all these results were derived from general Chinese population, none of the studies specifically investigated hrHPV genotyping in women with cervical precancerous/cancerous lesions. Our current study is the one of the largest to investigate hrHPV genotype distribution among women with CIN2/3, showing the top three most common types were 
HPV16 (59.3\%), HPV58 (14.4\%) and HPV33 (10.0\%), while HPV18 was in the $6^{\text {th }}$ position $(6.0 \%)$. A recent study indicated that the prevalence of HPV58 was $22.1 \%$ and $21.1 \%$ among HIV-1 positive women with CIN2 in Burkina Faso and South Africa respectively, and $23.1 \%$ and $15.1 \%$ among women with CIN3 and above lesions. ${ }^{40}$

In China, the development and application of HPV vaccines still face many challenges. Our study demonstrated that, even though HPV16 was the predominant type of HPV among Chinese women with CIN2/3, the following most common HPV types were not exactly the same as those found in other countries or regions. These data may provide a basis for formulating cervical cancer prevention strategies in China.

On April 24, 2014, the US Food and Drug Administration (FDA) approved a proposal by Roche Molecular Systems, (HPV) test as an alternate primary cervical cancer screening method for women aged 25 to 65 years. Current study showed $8 \%$ CIN2/3 patients had a negative HPV testing result before the histological diagnosis of high grade dysplasia, indicating the limitation of HPV testing as primary cervical cancer screening.

In summary, current study is the one of the largest case studies of HPV genotyping in Chinese women with histological diagnosis of CIN2/3. The knowledge of variable hrHPV genotypes in different countries and regiones may be important for the development and application of HPV vaccines.

\section{Acknowledgements}

The study was supported by the grant from the Special Public Welfare Industry Research of National Health and Family Planning Commission (Beijing, China) (201402010).

\section{Competing Interests}

The authors have declared that no competing interest exists.

\section{References}

[1] Torre LA, Bray F, Siegel RL, et al. Global cancer statistics, 2012. CA Cancer J Clin. 2015; 65(2): 87-108.

[2] Bray F, Ren JS, Masuyer E, Ferlay J. Global estimates of cancer prevalence for sites in the adult population in 2008. Int J Cancer. 2013; 132: 1133-1145.

[3] Shi JF, Qiao YL, Smith JS, et al. Epidemiology and prevention of human papillomavirus and cervical cancer in China and Mongolia. Vaccine. 2008; 26 Suppl 12:M53-59.

[4] Wang X, Liu K, Yuan F, et al. Analysis of both high incidence of esophageal and cervical cancer in Yangcheng County, Shanxi Province. China Cancer. 2011; (4):259-261.

[5] Walboomers JM, Jacobs MV, Manos MM, et al. Human papillomavirus is a necessary cause of invasive cervical cancer worldwide. J of Pathol.1999; 189:12-19.

[6] de Sanjose S, Diaz M, Castellsague X, et al. Worldwide prevalence and genotype distribution of cervical human papillomavirus DNA in women with normal cytology: a meta-analysis. The Lancet Infectious diseases.2007; 7:453-459.
[7] Clifford GM, Gallus S, Herrero R, et al. Worldwide distribution of human papillomavirus types in cytologically normal women in the International Agency for Research on Cancer HPV prevalence surveys: a pooled analysis. Lancet .2005; 366:991-998.

[8] Zhang R, Velicer C, Chen W, et al. Human papillomavirus genotype distribution in cervical intraepithelial neoplasia grades 1 or worse among 4215 Chinese women in population-based study. Cancer Epidemiol. 2013; 37(6):939-45.

[9] Zeng Z, Yang H, Li Z, et al. Prevalence and Genotype Distribution of HPV Infection in China: Analysis of 51,345 HPV Genotyping Results from China's Largest CAP Certified Laboratory. J Cancer. 2016; 7(9):1037-43.

[10] Giuliano AR, Kreimer AR, de Sanjose S. The beginning of the end: vaccine prevention of HPV-driven cancers. J Natl Cancer Inst. 2015; 107(6 ):djv128.

[11] Burchell AN, Winer RL, de Sanjosé S, et al. Chapter 6: Epidemiology and transmission dynamics of genital HPV infection. Vaccine.2006;24:S3/52-61.

[12] Sankaranarayanan R. HPV vaccination: The most pragmatic cervical cancer primary prevention strategy. Int J Gynaecol Obstet. 2015;131 Suppl 1:S33-35.

[13] Skinner SR, Apter D, De Carvalho N, et al. Human Expert review of vaccines. 2016; 15 (3):367-87.

[14] Tao P, Zheng W, Wang Y, Bian M. Sensitive HPV genotyping based on the flow-through hybridization and gene chip. J Biomed Biotechnol.2012, doi:10.1155/2012/938780.

[15] Xu XX, Zhou JS, Yuan SH, et al. Distribution of HPV genotype in invasive cervical carcinoma and cervical intraepithelial neoplasia in Zhejiang Province, Southeast China: establishing the baseline for surveillance. Int J Environ Res Public Health. 2015; 12(9): 10794-10805.

[16] Li N, Franceschi S, Howell-Jones R, et al. Human papillomavirus type distribution in 30,848 invasive cervical cancers worldwide: Variation by geographical region, histological type and year of publication. Int J Cancer. 2011; 128:927-935.

[17] Human papillomaviruses. In: IARC, Humans WHitEcoURt. Biological Agents. Volume $100 \mathrm{~B}$. A review of human carcinogens. IARC monographs on the evaluation of carcinogenic risks to humans / World Health Organization, International Agency for Research on Cancer 2012; 100:1-441.

[18] Bruni L, Diaz M, Castellsague X, et al. Cervical human papillomavirus prevalence in 5 continents: meta-analysis of 1 million women with normal cytological findings. J Infect Dis. 2010; 202:1789-1799.

[19] Castellsagué X, de Sanjosé S, Aguado KS. WHO/ICO Information Centre on HPV and Cervical Cancer. HPV and cervical cancer in the 2007 report. Vaccine. 2007; 25 Suppl 3:C1-230.

[20] Jing L, Zhong X, Zhong Z, et al. Prevalence of human papillomavirus infection in Guangdong province, China: a population-based survey of 78,355 women. Sex Transm Dis. 2014; 41:732-738.

[21] Li C, Wu M, Wang J, et al. A population-based study on the risks of cervical lesion and human papillomavirus infection among women in Beijing, People's Republic of China. Cancer Epidemiol Biomarkers Prev.2010;19:2655-64.

[22] Cairenzhuoma, Zhang J, Wei C. Human papillomavirus infection in 26,622 women in Qinghai. Chin J Fam Plann. 2013; 21(12):812-816.

[23] Fernandes JV, Meissner RV, Carvalho MG, et al. Prevalence of human papillomavirus in archival samples obtained from patients with cervical pre-malignant and malignant lesions from Northeast Brazil. BMC Res Notes. 2010; 3(1):96.

[24] Zhao F, Li Na, Ma J, et al. Study of the association between human papillomavirus infection and cervical cancer in Xiangyuan County, Shanxi province. Chin J Epidemiol. 2001; 22 (5): 375-378

[25] Wang L, Li J, Chen L. Prevalence of human papillomavirus and its genotype among 1336 invasive cervical cancer patients in Hunan province, central south China. J Med Virol. 2015; 87:516-521.

[26] Dickson EL, Vogel RI, Bliss RL, et al. Multiple-type human papillomavirus (HPV) infections: across-sectional analysis of the prevalence of specific types in 309,000 women referred for HPV testing at the time of cervical cytology. Int J Gynecol Cancer. 2013 ;23(7):1295-1302.

[27] Fife KH, Cramer HM, Schroeder JM, et al. Detection of multiple human papillomavirus types in the lower genital tract correlates with cervical dysplasia. J Med Virol. 2001; 64(4) : 550-559.

[28] Munoz N, Bosch FX, de-Sanjose S, et al. Epidemiologic classification of human papillomavirus types associated with cervical cancer. N Engl J Med.2003; 348 (6): 518-527.

[29] An HJ, Cho NH, Lee SY, et al. Correlation of cervical carcinoma and precancerous lesions with human papillomavirus (HPV) genotypes detected with the HPV DNA chip microarray method. Cancer. 2003; 97( 7): 1672-1680.

[30] Wright TC Jr, Stoler MH, Behrens CM, Sharma A, Sharma K, Apple R. Interlaboratory variation in the performance of liquid-based cytology: insights from the ATHENA trial. Int J Cancer. 2014; 134(8):1835-1843.

[31] Kjær SK, Munk C, Junge J, Iftner T. Carcinogenic HPV prevalence and age-specific type distribution in40,382 women with normal cervical cytology, ASCUS/LSIL, HSIL, or cervical cancer: what is the potential for prevention? Cancer Causes Control. 2014 ;25(2):179-189.

[32] Peng Guan, Rebecca Howell-Jones, Ni Li, et al. Human papillomavirus types in 115,789 HPV-positive women: A meta-analysis from cervical infection to cancer. Int J Cancer. 2012; 131: 2349-2359.

[33] Human papillomaviruses. In: IARC, Humans WGotEoCRt: Biological agents. Volume 100 B. A review of human carcinogens. IARC monographs on the evaluation of carcinogenic risks to humans / World Health Organization, International Agency for Research on Cancer 2012; 100:1-441. 
[34] Castellsagué X, de Sanjosé S, Aguado KS, HPV WIICo, Cervical C: HPV and cervical cancer in the 2007report. Vaccine .2007; 25 Suppl 3:C1-230.

[35] Bosch FX, Burchell AN, Schiffman M, et al. Epidemiology and natural history of human papillomavirus infections and type-specific implications in cervical neoplasia. Vaccine. 2008; 26 Suppl10:K1-16.

[36] G M Clifford, S Gallus, R Herrero, et al. Worldwide distribution of human papillomavirus types in cytologically normal women in the International Agency for Research on Cancer HPV prevalence surveys: a pooled Analysis. Lancet. 2005;366: 991-998.

[37] Ding X, Liu Z, Su J, et al. Human papillomavirus type-specific prevalence in women referred for colposcopic examination in Beijing. J Med Virol. 2014; 86:1937-43.

[38] Chih HJ, Lee AH, Colville L, et al. A review of dietary prevention of human papillomavirus-related infection of the cervix and cervical intraepithelial neoplasia. Nutr Cancer. 2013; 65(3):317-28.

[39] Zhao Y, Zhao F, Hu S, et al. Multi-center cross-sectional study on type-specific human papillomavirus infection among Chinese women. Chin J Epidemiol. 2015; 36(12):1351-1356

[40] Kelly HA, Ngou J, Chikandiwa A, et al. Associations of Human Papillomavirus (HPV) genotypes with high-grade cervical neoplasia (CIN2+) in a cohort of women living with HIV in Burkina Faso and South Africa. PLoS One. 2017; 12(3):e0174117. 